\title{
Exercise Testing and Stress Imaging in Aortic Valve Disease
}

\section{Luc A. Pierard, MD, PhD, FESC, FACC Raluca Dulgheru, MD}

\author{
Address \\ *Service de Cardiologie, CHU Sart Tilman, Domaine Universitaire du Sart Tilman \\ B35, 4000, Liege, Belgium \\ Email: lpierard@chu.ulg.ac.be
}

(C) Springer Science+Business Media New York 2017

This article is part of the Topical Collection on Valvular Heart Disease

The online version of this article (doi:10.1007/s11936-017-0551-5)

contains supplementary material, which is available to authorized users.

Keywords Aortic valve disease - Aortic stenosis - Left ventricular ejection fraction

\section{Opinion statement}

Aortic valve disease and especially aortic stenosis (AS) is a growing cardiac pathology. Aortic valve replacement (AVR) is still the only treatment with proven benefit on survival in symptomatic patients and in patients with a left ventricular ejection fraction (LVEF) $<50 \%$. The benefit of prophylactic AVR in asymptomatic patients is still unproven. Once symptoms develop, the prognosis worsens. Exercise testing has emerged as a tool to unmask the "pseudo-asymptomatic" patients with AS (those without self-reporting symptoms), to link "exercise induced dyspnea" more confidently and more objectively to aortic valve disease and to allow for a safe "watchful waiting strategy" in "pseudo-symptomatic" patients (those with dyspnea unrelated to aortic valve disease). In cases in which exercise testing is unable to link dyspnea to aortic valve disease, exercise stress echocardiography and cardiopulmonary exercise testing may be helpful. Whatever the results of exercise testing with regard to symptom development, an increase in mean aortic valve pressure gradient $>18-20 \mathrm{mmHg}$ was associated with an increased risk of cardiac related events in severe AS patients (class IIb indication for AVR in the ESC guidelines). The decrease in LVEF during exercise as well as the development of exercise induced pulmonary hypertension, as revealed by exercise stress echocardiography, may be also useful in the risk stratification of these asymptomatic patients with severe AS. Data on the role of exercise echocardiography in asymptomatic severe aortic regurgitation patients is still scarce and further studies are needed. It seems that an exercise induced decrease in LVEF by $5 \%$ may be a better predictor of LV systolic dysfunction after AVR in asymptomatic patients or in patients with minimal symptoms. Exercise testing and exercise echocardiography are safe in the asymptomatic patients with aortic disease, provide useful clinical information that may help in risk assessment of these complicated patients and their use should be encouraged especially in heart valve clinics. 
Significant aortic valve disease (more than moderate aortic stenosis or/and aortic regurgitation) affects between 2.8 and $4.8 \%$ of adults older than 75 years in the USA [1]. Aortic valve replacement (AVR) is the only treatment with proven benefit on survival in symptomatic patients (symptoms reported spontaneously or developed during exercise testing) and in patients with a left ventricular ejection fraction (LVEF) $<50 \%[2,3]$.

When the presenting symptom in patients with aortic stenosis (AS) is "exercise induced dyspnea," it is challenging to establish whether the symptom is clearly related to the valve disease. On the other hand, elderly patients with severe AS tend to unconsciously lower their level of physical activity to avoid symptoms ("pseudo-asymptomatic" patients) and therefore, tend to report symptoms too late. Some patients deny symptoms. Exercise testing has emerged as a tool to unmask the "pseudo-asymptomatic" patients with severe AS, and to link "exercise induced dyspnea" to the severity of valve disease. Still, even in the era of exercise testing, linking accurately exercise induced dyspnea to valve disease can be difficult, especially in patients with other comorbidities, such as obesity, chronic pulmonary obstructive disease, and physical deconditioning. Even in the absence of comorbidities, self-reported exercise induced dyspnea may be subjective, some patients (i.e., sedentary patients) reporting dyspnea during physical activities even when valve disease is only moderate. If clear evidence in favor of early prophylactic AVR in truly asymptomatic severe AS were available, exercise testing would be used only to detect early "pseudo-asymptomatic" patients, since once symptoms have developed, the risk of sudden death and the surgical risk are both increasing. However, the controversy of "early prophylactic AVR" in truly asymptomatic severe AS is not yet solved. Therefore, exercise testing in severe AS patients is performed to unmask "pseudo-asymptomatic patients" as well as to allow a safe "watchful waiting" strategy in "pseudo-symptomatic" patients (patients with severe AS but with dyspnea unrelated to valve disease). When exercise testing does not reveal symptoms, exercise stress echocardiography (ESE) and cardiopulmonary exercise testing (CPET) may be helpful for risk stratification of asymptomatic patients. This current review will cover shortly what is already established regarding exercise testing and exercise imaging in aortic valve disease, but will also give an update on what is new in this setting. Exercise testing in AS will be covered first, leaving at the end the less studied topic of exercise testing in aortic regurgitation (AR).

\section{Current guideline recommendations on exercise testing}

Exercise testing has been advocated by the ESC guidelines on the management of valvular heart disease to "unmask the objective occurrence of symptoms in patients who claim to be asymptomatic or have doubtful symptoms." [3]. No other specific recommendations are made regarding exercise testing and severe AR in the 2012 ESC guidelines [3]. In patients with severe AS specifically, the ESC guidelines emphasize that exercise testing "is recommended in physically active patients for unmasking symptoms and in the risk stratification of asymptomatic [ones]" [3].

In the 2014 ACC/AHA guidelines, exercise testing receives an "overall" class IIa indication (reasonable in selected patients) for the management of patients with asymptomatic valvular heart disease to "(1) confirm the absence of symptoms, or (2) assess the hemodynamic response to exercise, or (3) determine prognosis" [2]. With regard to severe AS, the ACC/AHA guidelines give a class IIa indication for exercise testing in asymptomatic patients with high gradient AS (stage C), with the purpose to confirm the absence of symptoms, while no specific class indication is made in patients with severe AR, in whom, guidelines state that "exercise stress testing can be used to assess symptomatic status and 
functional capacity and is helpful in confirming patients' reports that they have no symptoms with daily life activities and in assessing objective exercise capacity and symptom status in those with equivocal symptoms." [2].

Overall, because of the paucity of convincing data available up to now, both the ESC and the ACC/AHA guidelines give no specific class indication for exercise testing in asymptomatic patients with severe $A R$, nor a specific class indication for AVR if symptoms have been unmasked by exercise testing.

For severe AS, there is a class I indication for AVR in patients who develop symptoms during an exercise test in both guidelines. Moreover, the ESC guidelines emphasize that symptom development during exercise testing should be clearly related to AS. A class IIa indication for AVR is present in both guidelines if the patient has a drop in blood pressure values below baseline with exercise testing. However, a slight difference exists between the two guidelines with the American guideline including "decreased exercise tolerance" into the definition of "abnormal exercise test," and therefore as a class IIa indication for AVR, while for the European guidelines only the drop in blood pressure is considered as an abnormal response to exercise testing $[2,3]$.

To note, ventricular arrhythmias during exercise $[4,5], \geq 2 \mathrm{~mm}$ ST segment depression $[4,6]$, less than $20 \mathrm{mmHg}$ increase in blood pressure values during exercise $[4,6]$, mean gradient increase of $>18-20 \mathrm{mmHg}[5,6]$ with exercise and exercise induced fall in LVEF [7] have been shown to be predictive of spontaneous symptoms onset [4-6], symptom-driven AVR [5, 6], and sudden cardiac death $[5,6]$. However, due to the relatively weak evidence available, most of these abnormal findings during exercise testing carry no indication for AVR, with the exception of an increase in mean gradient with exercise of $>20 \mathrm{mmHg}$ which is a class IIb indication for AVR only in the ESC guidelines.

\section{Clinical practice issues/problems}

Angina, syncope, pre-syncope during exercise are easy to recognize and link to valve disease. However, they are rather rare in the AS population undergoing exercise testing. Virtually, all patients with severe AS performing an exercise test will end up being dyspneic at the end of the test. What is challenging is to recognize what represents "pathological dyspnea", and link it to valve disease. One would argue that dyspnea developing in the early stages of exercise testing is to be considered pathological and very likely related to valve disease. Much more challenging is to judge whether dyspnea arising in the mid-late stages of an exercise test is to be considered pathological, especially in untrained/ deconditioned subjects or subjects with multiple comorbidities. With this aim, ESE and CPET have been studied in patients with severe AS.

\section{What is known}

Whatever the results of the exercise test with regard to symptom development, an increase in mean aortic pressure gradient by $>18$ to $20 \mathrm{mmHg}$ during exercise was associated with an increased risk of cardiac-related events in 2 studies [5, 6].Our group was the first to report, in a series including 69 consecutive patients with asymptomatic severe AS and normal LV function that a significant increase 
in mean gradient with exercise may be a marker of worse prognosis [6]. Patients with marked exercise-induced increase in mean aortic pressure gradient had significantly worse cardiac event-free survival (around $80 \%$ of events at 2-year follow-up). Although patients who experienced an event during the follow-up had no significant difference in mean aortic pressure gradient at rest, as compared to those without an event $(41 \pm 12 \mathrm{mmHg}$ vs. $38 \pm 9 \mathrm{mmHg})$, they exhibited a significantly higher exercise-induced increase in mean gradient $(\Delta 23 \pm 8 \mathrm{mmHg}$ vs. $\Delta 12 \pm 7 \mathrm{mmHg}$ ). This parameter provided incremental prognostic value over resting echocardiographic data and exercise electrocardiogram. Similar results have been found in a multi-centric study which enrolled 186 "truly" asymptomatic patients (i.e., without abnormality during an exercise test) with at least moderate AS (AVA $<1.5 \mathrm{~cm}^{2}$ ) [5]. In this study, an exercise-induced increase in mean aortic pressure gradient $>20 \mathrm{mmHg}$ was independently associated with a 3.8-fold increase in risk of cardiac events, regardless of age, exercise LV ejection fraction, or resting mean aortic pressure gradient. Interestingly, this marked increase in mean gradient during exercise (i.e., $>18$ to $20 \mathrm{mmHg}$ ) was encountered in 35 and $21 \%$, respectively, of the studied population in these two studies $[5,6]$. This "pathological" increase in pressure gradient during exercise may reflect the presence of either a more severe AS (the more severe the stenosis at rest, the higher the increase in gradient for a given flow rate during exercise) and/or a noncompliant, rigid aortic valve (no or minimal improvement of aortic valve orifice opening during exercise).The exercise-induced changes in mean aortic pressure gradient should, however, be analyzed in the light of the exercise-induced changes in LV stroke volume. Indeed, a marked increase in LV stroke volume in response to exercise may directly influence the aortic mean pressure gradient, even in the presence of a compliant aortic valve with a certain degree of opening reserve. In contrast, in highly calcified aortic stenosis, without improvement in aortic valve area during exercise, mild increase in LV stroke volume may produce as well significant changes in mean aortic pressure gradient.

Of similar clinical interest and equally feasible with exercise echocardiography is the assessment of LV function during exercise. It has been shown that patients with a decrease or a small increase in LV ejection fraction during exercise are more likely to exhibit an abnormal response to exercise and cardiac-related events during follow-up $[7,8]$. In the study by Marechaux et al. [7], absence of $\mathrm{LV}$ contractile reserve defined as no increase or reduction of $\mathrm{LV}$ ejection fraction with exercise was associated with more frequent abnormalities, with development of symptoms during exercise and with markedly reduced midterm cardiac event-free survival (around $40 \%$ at 2-year follow-up). Furthermore, abnormal response to exercise (excessive symptoms, fall or $<20 \mathrm{mmHg}$ increase in systolic blood pressure, and $>2 \mathrm{~mm}$ ST-segment depression) was associated with the decrease/mild increase of LV ejection fraction during exercise [9]. With 2D speckle tracking analysis, Donal et al. reported a threshold of $-1.4 \%$ in exercise-induced changes in LV global longitudinal strain as an accurate marker of the presence of LV contractile reserve [8]. However, it should be emphasized that these studies are based on relatively small sample sizes and the cut-off values were not validated against patients' outcome.

In severe AS, elevated SPAP and the presence of pulmonary hypertension (PHT) (SPAP $>50 \mathrm{mmHg}$ ) at rest seem to be associated with a poor prognosis $[10,11]$ and a higher mortality rate after valve replacement [12]. Moreover, 
elevated SPAP and PHT at rest represent an independent predictor of hospital mortality and post-operative major adverse cardiovascular and cerebrovascular events [13]. The inconvenience is that, although an easy to assess parameter by resting echocardiography, when present, PHT is often associated with the presence of symptoms, which limits its usefulness for clinical decision making. In contrast, exercise PHT is generally considered as a predictor of occurrence of resting PHT during the follow-up, development of symptoms, and/or outcome in various cardiac diseases, including valvular heart disease [14, 15]. We have previously reported the results of 105 "truly" asymptomatic consecutive patients with severe AS in whom exercise echocardiography was performed to identify the changes in SPAP during exercise [16]. Whereas only $6 \%$ of the population exhibited resting PHT (SPAP $>50 \mathrm{mmHg}$ ), 55\% of patients developed exercise PHT (SPAP $>60 \mathrm{mmHg}$ ). Exercise PHT was mainly determined by resting SPAP and male gender, but also by exercise parameters of diastolic dysfunction (exercise indexed LV end-diastolic volume, exercise $e^{\prime}$-wave velocity and exercise-induced changes in indexed left atrial area). Moreover, exercise PHT was independently associated with a twofold increase in risk of cardiac event at 3-year follow-up. All 7 cardiovascular deaths (3 sudden deaths and 4 deaths following heart failure requiring hospitalization) reported in this series, occurred in the group of patients with exercise PHT, whereas only 1 patient died from the group with resting PHT. Practically, the measurement of SPAP throughout the exercise could provide useful additive information. However, the dynamic changes in SPAP and the occurrence of exercise PHT should be cautiously interpreted and analyzed in light of age, exercise load, and changes in systemic blood pressure and in cardiac output.

Evaluation of the respiratory gas-exchange during exercise and the measurement of peak maximal oxygen uptake (VO2) may be of interest in AS patients. In a recent bi-centric study, we have shown that asymptomatic patients with AS might have a reduced maximal exercise capacity, even in the absence of LV dysfunction or abnormal exercise response (in terms of symptoms, blood pressure, or electrical changes) [17]. Indeed, close to one-half of our patients had markedly reduced $(<84 \%)$ age- and sex-predicted peak VO2. Furthermore, the main determinant of impaired maximal exercise capacity was elevated global LV hemodynamic afterload (i.e., valvulo-arterial impedance, a surrogate of both valvular and arterial load faced by the LV). Therefore, the measurement of peak $\mathrm{VO} 2$ may provide an objective parameter allowing a better assessment of the symptomatic status and unmasking patients who deny symptoms or adapt their life-style to their condition. However, the relationship between decreased maximal exercise capacity in asymptomatic AS patients and outcome requires clarification and further studies.

\section{What is new}

Three interesting studies, two of them using ESE and hemodynamic invasive measurements in symptomatic patients with low or high gradient AS, respectively, and another study using post treadmill exercise echocardiography have been published in the past year with interesting results $[18 \bullet \bullet, 19,20 \bullet \bullet]$.

All of these studies reinforce the finding that exercise testing is safe in asymptomatic patients with severe AS, with none of the studies reporting 
untoward events during or after the exercise test in this high risk population. Lumley et al. and Perez del Villar et al. shown that supine bicycle exercise testing during cardiac catheterization in patients with severe AS studied prior to AVR, and thus, symptomatic, is feasible and safe $[18 \bullet \bullet, 20 \bullet \bullet$. The study of Perez del Villar et al. is also interesting from the safety point of view because it enrolled

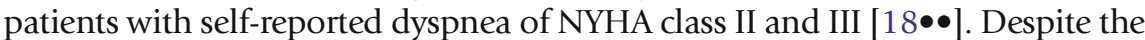
fact that NYHA class II-III patients have been enrolled, no adverse events have been recorded. Hence, ESE is safe, even in mildly symptomatic patients with severe AS, in experienced hands. In our view based on our experience with ESE, this may be related to the fact that ESE is a highly controlled type of exercise testing with continuous patient status monitoring including heart rate, blood pressure response, evaluation of LV function response, and continuous monitoring of systolic pulmonary pressure. In this setting, the physician will immediately stop exercise testing in a dyspneic patient in whom new wall motion abnormalities and/or LV systolic dysfunction and/or exercise-induced pulmonary hypertension are observed. In our opinion, this is one of the important advantages of ESE over post exercise echocardiography, and this may partially explain the low adverse event rate with ESE in mildly symptomatic patients with

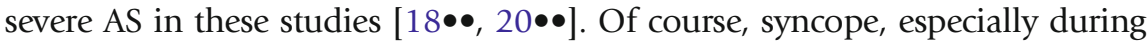
exercise or typical angina remain contraindications for exercise stress testing.

Masri et al. retrospectively analyzed the data from 533 prospectively enrolled patients with asymptomatic severe AS with preserved LVEF (i.e. LVEF $>50 \%$ ), defined by an indexed AVA of $<0.6 \mathrm{~cm}^{2} / \mathrm{m}^{2}$ who underwent treadmill exercise testing [19]. Post exercise Doppler echocardiography data have been collected in some of the patients and in 281patients (53\%) post exercise mean gradient was available. The first interesting finding of this study is that of all patients considered "asymptomatic" by history taking, only $50 \%$ were able to carry on the exercise treadmill to $>100 \%$ (excellent exercise capacity), $24 \%$ achieved between 85 to $100 \%$ and $26 \%$ achieved $<85 \%$ age-sex-predicted METs. Almost $19 \%$ of all patients developed symptoms (dyspnea, angina, dizziness, abnormal BP or arrhythmias) and patients who achieved $<85 \%$ age and sex-predicted METs were more likely to develop symptoms. To note, severity of AS in this study was defined based on an AVA $<0.6 \mathrm{~cm}^{2} / \mathrm{m}^{2}$ and some of the patients had a BMI $>25 \mathrm{~kg} / \mathrm{m}^{2}$ which in combination with the fact that in the whole cohort the average peak velocity was $<4 \mathrm{~m} / \mathrm{s}$ may imply that some patients had moderate to severe forms of AS. If all patients in the spectrum of moderate to severe AS were part of the group with excellent/preserved exercise capacity, it means that in the actual population with severe AS a lower percentage of patients would have been able to achieve $>100 \%$ age sexpredicted METs.

The main finding of the study was that lower \% age and sex-predicted METs and slower heart rate recovery (defined as the drop in heart rate from peak to 1 min post exercise) along with a higher STS score were associated with higher long-term mortality [19]. As opposed to other studies [5, 6], in the subgroup of 281 patients in whom post exercise stress mean gradient was available, only $17 \%$ of patients had $>20 \mathrm{mmHg}$ increase in gradient after exercise and this increase was not associated with longer-term mortality. To understand the discrepancies with previous reports and the clinical implications of these results, a careful review of the methodology used in the present study is necessary. First, only $17 \%$ of the 281 patients had an increase in mean gradient with exercise of 
$>20 \mathrm{mmHg}$. In the whole cohort, the mean gradient after exercise was only $44 \pm 12 \mathrm{mmHg}$, as opposed to what was reported in previous studies. If only the study of Lancellotti et al. is considered for comparison [6], since it included patients with severe AS (i.e. AVA $<1.0 \mathrm{~cm}^{2}$ ), the mean gradient during exercise was considerably higher as compared to the study of Masri et al. $(53 \pm 15 \mathrm{mmHg}$ in patients without events at follow-up and $60 \pm 11 \mathrm{mmHg}$ in those with events during follow-up vs. $44 \pm 12 \mathrm{mmHg}$ in the study of Masri et al.) [6, 19]. In our view, this difference relates to the fact that in the study of Masri et al., mean gradient was recorded during the recovery period after exercise and not at peak exercise. In our experience, the increase in gradient with exercise may be shortlived after exercise cessation. Moreover, from the methodology section, it appears that immediately after exercise the echo exam focus was set on regional wall motion abnormalities for evaluation of ischemia and peak right ventricular systolic pressure, while transaortic velocity and gradients were evaluated later in the time course of the evaluation. Interestingly, post exercise right ventricular systolic pressure was similar in patients with age and sex-predicted METs $\geq 85$ vs. $<85 \%$, although patients with age and sex-predicted METs $<85 \%$ were those more likely to present with symptoms during exercise, again a slightly discordant result as compared to studies looking at right ventricular systolic pressure assessment during exercise [16]. Close scrutiny of the data shows that some of the patients achieving $>85 \%$ of age-sex predicted METs did, indeed, develop symptoms during treadmill exercise testing, and there is an open question whether some of the patients developing symptoms were also the ones who have reached more than $100 \%$ of age and sex-predicted METs. Interesting data on AS population come also from the study of Perez del Villar et al., in lowgradient AS patients (20 patients with AS, LVEF $>50 \%$, AVA $<1.0 \mathrm{~cm}^{2}$ or AVA $<0.6 \mathrm{~cm}^{2} / \mathrm{m}^{2}$ and a mean gradient $<40 \mathrm{mmHg}$ ) who aimed to cast a light into its complicated pathophysiology with the help of combined exercise testing (during exercise echocardiography and during exercise catheterization). This study proves that in the correct setting, not only Doppler data can be assessed during exercise echocardiography but also hemodynamic measurements in a safe way, even in patients with mild symptoms (class II NYHA dyspnea in 19 patients, 1 patient with class III). Fifty percent of the studied population had a low-flow low-gradient AS, $\left(\mathrm{SVi}<35 \mathrm{ml} / \mathrm{m}^{2}\right)$ while the other half had normal-flow low-gradient AS (mean gradient $<40 \mathrm{mmHg}$ but $\mathrm{SVi} \geq 35 \mathrm{ml} / \mathrm{m}^{2}$ ). Symptom development during exercise was not reported in this study, although authors do emphasize that exercise was symptom-limited. No patient had a drop in blood pressure with exercise, or an increase in systolic blood pressure of less than $20 \mathrm{mmHg}$. Interestingly, by invasive hemodynamic measurements, $80 \%$ of patients developed increased left atrial pressure (PCWP $>16 \mathrm{mmHg}$ ). It would have been interesting to report the percentage of patients with an increase in left atrial pressure with exercise who stopped their exercise due to dyspnea and the agreement between invasively measured systolic pulmonary artery pressure and non-invasive Doppler method during exercise. No information was provided regarding the tissue Doppler parameter $\mathrm{E} / \mathrm{e}^{\prime}$ ratio considered a surrogate marker of elevated LV filling pressure with exercise. However, meaningful information can be retained from this study: (1) the more rapid the increase in capillary wedge pressure during exercise, the lower the maximal exercise capacity; (2) the best independent predictors of the increase in PCWP 
were the capacity to increase aortic valve compliance and the capacity to increase arterial compliance, but no rest echocardiographic parameters of valve stenosis severity or LV function; and (3) the patients with the greatest limitation of maximal exercise capacity had stiffer valves. However, type 2 error cannot be ruled out in such a small subject sample. The most important message of the study was that stenotic valves at rest may still have an "opening reserve" which in turn determines the degree of functional impairment in an individual patient during exercise.

The third study on exercise testing and AS that is worth mentioning is the study of Lumley et al. [20••]. This study has shown that invasive coronary physiological evaluation can be safely performed during exercise and hyperemia in patients with AS and that myocardial ischemia in AS is not related to microvascular disease, but rather to abnormal cardiac-coronary coupling. This was an elegant study that was performed during exercise catheterization in 22 patients with high gradient severe AS referred for surgery and compared to 38 healthy controls. Refined analysis of coronary blood flow using a dual pressure and Doppler sensor-tipped intracoronary wire was performed to assess coronary pressure and flow velocity and to derive information on microvascular resistance throughout the cardiac cycle, at rest, during exercise and also during hyperemic state. AS patients and 17 consecutive control patients were also examined with exercise echocardiography. The main findings of the study were (1) the mechanisms of coronary blood flow increase during exercise are fundamentally different from adenosine induced hyperemia; (2) symptomatic patients with severe AS failed to adapt their coronary blood flow to the increase in cardiac work, thus making the myocardium more vulnerable to ischemia; (3) minimum microvascular resistance in severe AS was not different from controls, supporting the hypothesis that microvascular disease is not a factor of reduced coronary flow reserve; (4) although the efficiency of the healthy heart improved during exercise and hyperemia due to an increase in the contribution of waves that accelerate coronary flow, the opposite was observed in severe AS due to an increase in the contribution of waves that decelerate flow. In the light of this elegant study, one may conclude that abnormal cardiac-coronary coupling (abnormal coronary pressure-flow physiology with exaggerated systolic pressure-flow deceleration waves together with a reduced diastolic perfusion time) is very likely to be responsible for myocardial ischemia in symptomatic patients with severe high gradient AS. However, one may need to bear in mind that these results may be applicable only to patients with "pure" forms of high gradient AS, since authors have excluded patients without important comorbidities, with coronary artery disease and LVEF $<50 \%$. Nonetheless, the findings of this study are helpful to understand that development of wall motion abnormalities during exercise testing in patients with severe AS may not reflect flow obstructive coronary artery disease (Fig. 1).

\section{Exercise testing and exercise stress imaging in aortic regurgitation}

Exercise testing is reasonable in asymptomatic patients with severe AR, but no class indication can be given in the clinical practice guidelines, since there are 


\section{a}

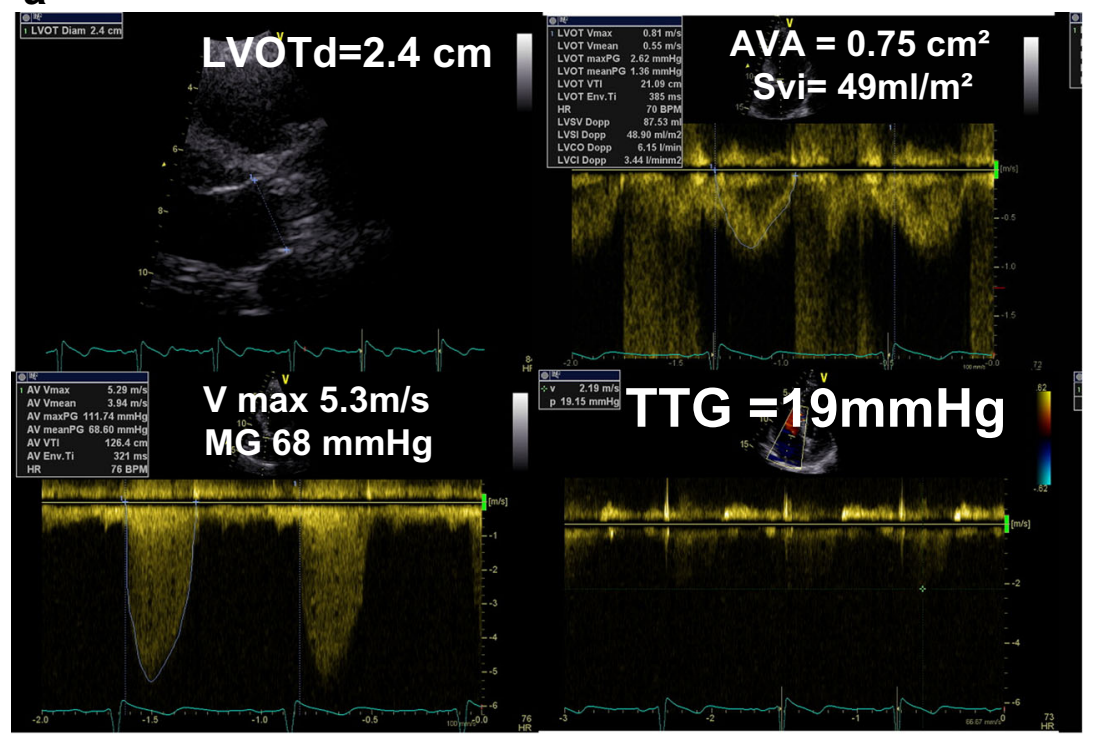

b

Rest

Low level exercise

Peak exercise

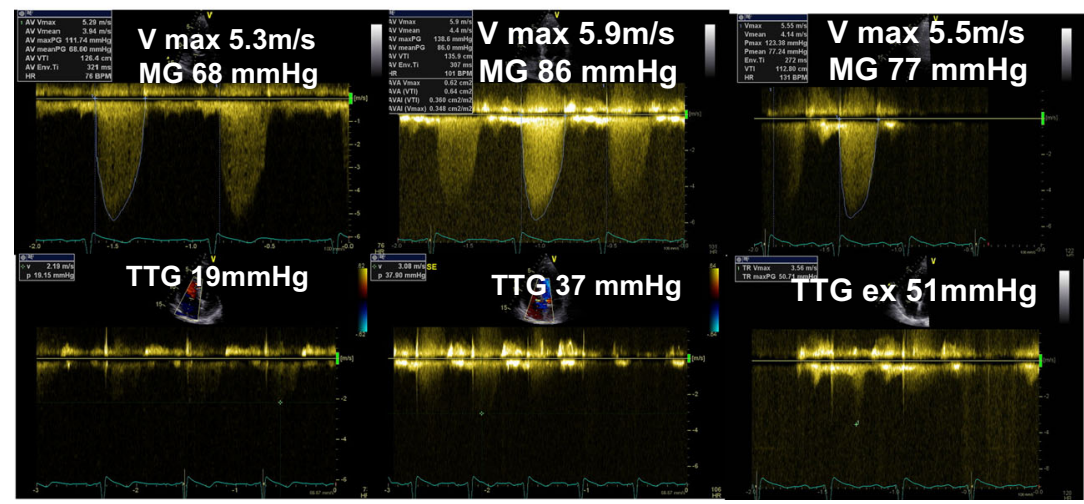

Fig. 1. a Top. b bottom. A 75-year-old active and asymptomatic patient with severe AS presented for evaluation in our heart valve clinic. High gradient severe AS with preserved LVEF has been confirmed (a). There is a $20-\mathrm{mmHg}$ increase in mean gradient and no significant increase in systolic pulmonary artery pressure in the first step of the exercise. At peak stress exercise, there is a decrease in mean transaortic gradient, paralleled by the development of new wall motion abnormalities (see Movie 1) and increase in pulmonary artery pressure. Importantly, systolic blood pressure increased less than $20 \mathrm{mmHg}$ during exercise and coronary angiography did not show any significant stenosis of the coronary arteries. LVOTd left ventricular outflow tract diameter, AVA aortic valve area by continuity equation, Svi stroke volume index, $V$ max maximal transvalvular velocity, MG mean transaortic gradient, $T T G$ systolic trans-tricuspid gradient, $E / e^{\prime}$ the ration between E-peak early diastolic wave velocity of the trans-mitral diastolic flow and the early peak diastolic velocity measured at the medial mitral annulus with tissue Doppler.

few studies that have addressed this issue [21, 22]. When exercise testing is performed together with echocardiographic examination, the focus should be set on the evaluation of LV function and pulmonary artery pressure estimation with Doppler. Limited data support the use of exercise echocardiography in asymptomatic AR. Wahi et al. have reported that contractile reserve as assessed with exercise echocardiography (decrease in LVEF by $5 \%$ with exercise) was a better predictor of LV systolic dysfunction after AVR than resting indices of LV 
function. This was studied in asymptomatic patients or patients with minimal symptoms [21].

Newer studies on aortic valve regurgitation during exercise testing remain scarce. Broch et al. have investigated 66 asymptomatic patients with moderate to severe aortic regurgitation, preserved LVEF (i.e. LVEF > 50\%) and without marked LV dilatation (i.e. end-diastolic LV diameter $<70 \mathrm{~mm}$ or end-systolic LV diameter $<50 \mathrm{~mm}$ ) who performed an exercise stress treadmill test with measurement of peak oxygen consumption [23]. In this cohort, high oxygen consumption was associated with the presence of a large end-diastolic LV volume, as assessed by CMR, a low resting heart rate and higher levels of circulating natriuretic peptides, while aortic regurgitation severity was not predictive of poorer maximal exercise capacity. The authors concluded that these patients have a fairly good maximal exercise capacity (mean peak $\mathrm{VO} 2$ in the cohort $25.8 \pm 8.9 \mathrm{ml} / \mathrm{kg}$ ) and that $\mathrm{LV}$ dilatation is mainly an adaptative mechanism. However, an increase in the level of natriuretic peptides seems to be a more sensitive marker of decreased exercise capacity in such patients. The influence of these parameters on patients outcome has not been reported merit further investigation.

\section{Conclusion and future directions}

The use of exercise testing in aortic valve disease is safe in asymptomatic patients and should be encouraged especially in heart valve clinics in experienced hands to understand the complex response of the left ventricle during exercise. The combination of hemodynamic assessment and imaging has been shown to better stratify risk. Large-scale studies are mandatory to fully validate ESE in the setting of aortic valve diseases.

\section{Compliance with Ethical Standards}

\section{Conflict of Interest}

Luc A. Pierard and Raluca Dulgheru each declare no potential conflicts of interest.

Human and Animal Rights and Informed Consent

This article does not contain any studies with human or animal subjects performed by any of the authors.

\section{References and Recommended Reading}

Papers of particular interest, published recently, have been

highlighted as:

- Of importance

- Of major importance

1. Go AS, Mozaffarian D, Roger VL, et al. Heart disease and stroke statistics-2014 update: a report from the American Heart Association. Circulation. 2014;129:e28-e292.

2. Nishimura RA, Otto CM, Bonow RO, et al. 2014 AHA/ ACC guideline for the management of patients with valvular heart disease: executive summary: a report of the American College of Cardiology/American Heart 
Association task force on practice guidelines. J Am Coll Cardiol. 2014;63:2438-88.

3. Vahanian A, Alfieri O, Andreotti F, et al. Guidelines on the management of valvular heart disease (version 2012). Eur Heart J. 2012;33:2451-96.

4. Amato MC, Moffa PJ, Werner KE, Ramires JA. Treatment decision in asymptomatic aortic valve stenosis: role of exercise testing. Heart. 2001;86:381-6.

5. Marechaux S, Hachicha Z, Bellouin A, et al. Usefulness of exercise-stress echocardiography for risk stratification of true asymptomatic patients with aortic valve stenosis. Eur Heart J. 2010;31:1390-7.

6. Lancellotti P, Lebois F, Simon M, et al. Prognostic importance of quantitative exercise Doppler echocardiography in asymptomatic valvular aortic stenosis. Circulation. 2005; 112:I377-82.

First study to look at the increase in mean transaortic gradient during exercise stress echocardiography in patients with severe aortic stenosis.

7. Marechaux S, Ennezat PV, LeJemtel TH, et al. Left ventricular response to exercise in aortic stenosis: an exercise echocardiographic study. Echocardiography. 2007;24:955-9.

8. Donal E, Thebault C, O'Connor K, et al. Impact of aortic stenosis on longitudinal myocardial deformation during exercise. Eur J Echocardiogr. 2011;12:23541.

9. Lancellotti P, Karsera D, Tumminello G, Lebois F, Pierard LA. Determinants of an abnormal response to exercise in patients with asymptomatic valvular aortic stenosis. Eur J Echocardiogr. 2008;9(3):338-43.

10. Cooper R, Ghali J, Simmons BE, Castaner A. Elevated pulmonary artery pressure. An independent predictor of mortality. Chest. 1991;99:112-20.

11. McHenry MM, Rice J, Matlof HJ, Flamm MD Jr. Pulmonary hypertension and sudden death in aortic stenosis. Br Heart J. 1979;41:463-7.

12. Copeland JG, Griepp RB, Stinson EB, Shumway NE. Long-term follow-up after isolated aortic valve replacement. J Thorac Cardiovasc Surg. 1977;74:875-89.

13. Carnero-Alcazar M, Reguillo-Lacruz F, Alswies A, et al. Short- and mid-term results for aortic valve replacement in octogenarians. Interact Cardiovasc Thorac Surg. 2010;10:549-54.

14. Magne J, Lancellotti P, Pierard LA. Exercise pulmonary hypertension in asymptomatic degenerative mitral regurgitation. Circulation. 2010;122:33-41.

15. Brochet E, Detaint D, Fondard O, et al. Early hemodynamic changes versus peak values: what is more useful to predict occurrence of dyspnea during stress echocardiography in patients with asymptomatic mitral stenosis? J Am Soc Echocardiogr. 2011;24:392-8.

16. Lancellotti P, Magne J, Donal E, et al. Determinants and prognostic significance of exercise pulmonary hypertension in asymptomatic severe aortic stenosis. Circulation. 2012;126:851-9.

17. Dulgheru R, Magne J, Capoulade R, et al. Impact of global hemodynamic load on exercise capacity in aortic stenosis. Int J Cardiol. 2013;168:2272-7.

18.• Perez Del Villar C, Yotti R, Espinosa MA et al. The functional significance of paradoxical low gradient aortic valve stenosis: hemodynamic findings during cardiopulmonary exercise testing. JACC Cardiovasc Imaging. 2017;10(1):29-39.

First study to show through a combined Doppler and hemodynamic approach that stenotic low gradient aortic valves may have a significant opening reserve during exercise that may explain, in part, the increase in capillary wedge pressure and exercise inadaptation.

19. Masri A, Goodman AL, Barr T et al. Predictors of longterm outcomes in asymptomatic patients with severe aortic stenosis and preserved left ventricular systolic function undergoing exercise echocardiography. Circ Cardiovasc Imaging. 2016;9(7). doi:10.1161/ CIRCIMAGING.116.004689.

20.• Lumley M, Williams R, Asrress KN, et al. Coronary physiology during exercise and vasodilation in the healthy heart and in severe aortic stenosis. J Am Coll Cardiol. 2016;68:688-97.

First study to perform an analysis of coronary arteries flow during bicycle exercise in patients with severe aortic stenosis which were symptomatic and to prove that coronary artery coupling is abnormal in these patients, making them possibly at risk for myocardial ischemia during exercise.

21. Wahi S, Haluska B, Pasquet A, et al. Exercise echocardiography predicts development of left ventricular dysfunction in medically and surgically treated patients with asymptomatic severe aortic regurgitation. Heart. 2000;84:606-14.

22. Lindsay J Jr, Silverman A, Van Voorhees LB, Nolan NG. Prognostic implications of left ventricular function during exercise in asymptomatic patients with aortic regurgitation. Angiology. 1987;38:386-92.

23. Broch K, Urheim S, Massey R, et al. Exercise capacity and peak oxygen consumption in asymptomatic patients with chronic aortic regurgitation. Int J Cardiol. 2016;223:688-92. 